\title{
Efficacy of tacrolimus for induction of remission in patients with moderate-to-severe ulcerative colitis: a systematic review and meta-analysis
}

\author{
Juan LASA and Pablo OLIVERA
}

Received 24/11/2016

Accepted 26/12/2016

ABSTRACT - Background - There is evidence that shows that calcineurin inhibitors may be useful for the treatment of severe ulcerative colitis. However, evidence regarding the efficacy of tacrolimus for remission induction in this setting is scarce. Objective - To develop a systematic review on the existing evidence regarding the clinical efficacy of tacrolimus for the induction of remission in patients with moderate-to-severe ulcerative colitis. Methods - A literature search was undertaken from 1966 to August 2016 using MEDLINE, Embase, LILACS and the Cochrane Library. The following MeSH terms were used: "Inflammatory Bowel Diseases" or "Ulcerative Colitis" and "Calcineurin Inhibitors" or "Tacrolimus" or "FK506". Studies performed in adult ulcerative colitis patients that evaluated the clinical efficacy of tacrolimus for the induction of remission were considered for revision. A meta-analysis was performed with those included studies that were also placebo-controlled and randomized. Clinical response as well as clinical remission and mucosal healing were evaluated. Results - Overall, 755 references were identified, from which 22 studies were finally included. Only two of them were randomized, placebo-controlled trials. A total of 172 patients were evaluated. A significantly lower risk of failure in clinical response was found for tacrolimus versus placebo [RR 0.58 (0.45-0.73)]; moreover, a lower risk of failure in the induction of remission was also found versus placebo [RR $0.91(0.82-1)]$. Conclusion - Tacrolimus seems to be a valid therapeutic alternative for the induction of remission in patients with moderate-to-severe ulcerative colitis.

HEADINGS - Inflammatory bowel diseases. Tacrolimus. Calcineurin inhibitors.

\section{INTRODUCTION}

Inflammatory Bowel Disease (IBD) is an immunological condition that carries a significant morbidity as well as mortality ${ }^{(1)}$. It has been classified as Crohn's Disease (CD) or Ulcerative Colitis (UC). Even though these entities share a common ground, they exhibit differences in terms of clinical presentation and therapeutic alternatives. During the last few years, there has been a significant development of scientific evidence showing the benefit of biological therapy - mainly anti-TNF $\alpha$ agents - in both scenarios ${ }^{(7)}$.

Nevertheless, approximately $30 \%$ of patients with moderateto-severe disease, whether they are CD or UC patients, do not respond to anti-TNF $\alpha$ therapy (primary failure) ${ }^{(3)}$ and a significant proportion of patients (13\% to $25 \%$ per year of treatment) may develop resistance and hence loss of efficacy to this type of treatment (secondary failure) ${ }^{(27,33)}$. This is why alternative therapeutic strategies are needed for these patients so that clinical conditions are improved and potentially serious complications can be avoided.

There is some evidence that show the efficacy of calcineurin inhibitors for the treatment of patients with severe $\mathrm{UC}^{(18)}$. Due to the effect that these drugs exert on calcineurin, they can inhibit the transcription of the Interleukin-2 gene - among others - which is necessary for the activation of T lympochytes ${ }^{(2)}$. As a consequence, although the quality of the available evidence is far from ideal, cyclosporine has been used in this clinical context; much less evidence is available in the $\mathrm{CD}$ scenario.
Bearing this in mind, a class-effect on moderate-to-severe UC could be inferred; consequently, the use of other calcineurin inhibitors such as tacrolimus has been proposed. Tacrolimus has the advantages of oral administration, a well-known safety profile and the previous experience of use in other clinical settings ${ }^{(3)}$. However, the evidence of the efficacy of its use in moderate-to-severe UC is scarce.

As a consequence, we sought to carry out a systematic review of the available evidence on this matter, with a meta-analysis considering randomized controlled trials.

\section{METHODS}

A literature search was carried out from 1966 to August 2016 using the following databases: MEDLINE, Embase, LILACS and The Cochrane Library. The search strategy included the following MeSH terms: "Inflammatory Bowel Disease" or "Ulcerative Colitis" and "Calcineurin Inhibitors" or "Tacrolimus" or "FK-506". Also, we reviewed the bibliographic references of the papers identified as potentially relevant. Additionally, we manually reviewed the abstracts of the Digestive Disease Week and United European Gastroenterology Week from 2010 to 2016.

The two authors performed bibliographic search in an independent manner. Potentially relevant abstracts were reviewed to check if they fulfilled inclusion criteria. These criteria were: a) studies that addressed the efficacy of tacrolimus in more than five UC patients; b) studies performed in adult population. For meta- 
analysis performance, studies which fulfilled the aforementioned criteria and were randomized controlled trials were included. PRISMA recommendations were followed for this purpose.

Authors' findings were then compared. If there was disagreement regarding the inclusion of a study, this would be discussed until consensus was reached. If data duplication was suspected, the authors of the studies would be contacted to exclude this situation.

The methodological quality of the included studies would be evaluated following the Evidence-Based Gastroenterology Sterring Group recommendations ${ }^{(29)}$. In addition, Jadas score was estimated for each study.

Outcome measures were: clinical response, clinical remission after induction treatment and, if available, mucosal healing rate.

Meta-analysis was performed using REVMAN software (Review Manager Version 5.1. Copenhagen: The Nordic Cochrane Center, The Cochrane Collaboration, 2011). Heterogeneity was estimated by means of I2 test and chi square test. If no significant heterogeneity was found, a fixed-effect model would be used for meta-analysis; otherwise, a random-effect model would be preferred. Outcome measures were described as Relative Risks (RR) with their corresponding 95\% Confidence Interval $(95 \% \mathrm{CI})$. Additionally, their corresponding Number Necessary to Treat (NNT) were calculated.

\section{RESULTS}

Overall, 755 potentially relevant citations were identified; 48 of them were furtherly chosen for analysis. Figure 1 shows the flow diagram which describes the reasons for citations exclusion. Finally, 22 studies $^{(4-6,9-17,20-25,28,30,32,33)}$ that fulfilled eligibility criteria were included. Only two studies were randomized controlled trials ${ }^{(24,25)}$ (Ogata 2006 and Ogata 2012); these were included for meta-analysis.

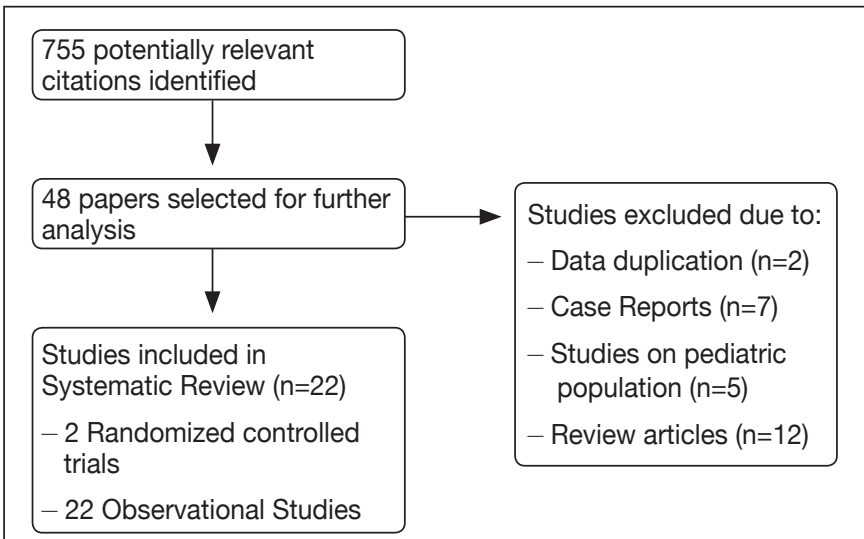

FIGURE 1. Flow-Chart showing the selection process of the studies included in the systematic review.

Table 1 describes the main features of the non-controlled studies that evaluated the efficacy of tacrolimus in patients with UC. The population included in these studies are rather similar - moderateto-severe UC patients which are steroid-dependent or steroidrefractory- but certain discrepancy was found in the way that clinical response and clinical remission were defined: some studies used Truelove Witts criteria, whereas others used Lichtiger score. Some minor differences in terms of follow up time were also found. It is worth mentioning that only one of these studies ${ }^{(14)}$ (Ikeya 2015) considered mucosal healing as an outcome. The tacrolimus dosage was adjusted to obtain a serum level between 10 to $15 \mathrm{ng} / \mathrm{mL}$. Overall, 609 patients were included in non-controlled studies: in many of these cases, colectomy was delayed but not avoided.

TABLE 1. Characteristics of observational studies included in the systematic review

\begin{tabular}{|c|c|c|c|c|c|c|c|c|}
\hline Author & Year & Country & $\begin{array}{l}\text { UC severity } \\
\text { and } \\
\text { distribution }\end{array}$ & $\begin{array}{c}\text { Remission } \\
\text { definition (and } \\
\text { time considered) }\end{array}$ & $\begin{array}{l}\text { Number } \\
\text { of } \\
\text { patients }\end{array}$ & Interventions & $\begin{array}{l}\text { Concomitant } \\
\text { treatments }\end{array}$ & Results \\
\hline Fellermann ${ }^{(10)}$ & 2002 & Germany & $\begin{array}{l}\text { Moderate-to- } \\
\text { severe colitis/ } \\
\text { steroid- } \\
\text { refractory }\end{array}$ & $\begin{array}{l}\text { Truelove-Witts } \\
\text { Score. Remission } \\
\text { evaluation in } 2 \\
\text { weeks. Follow up } \\
\text { for } 6 \text { months }\end{array}$ & 38 & $\begin{array}{l}\text { IV Tacrolimus } \\
(0.01-0.02 \mathrm{mg} / \mathrm{kg} / \\
\text { day) or P.O. (0.1- } \\
0.2 \mathrm{mg} / \mathrm{kg} / \text { day })\end{array}$ & $\begin{array}{l}\text { Antibiotics; } \\
\text { aminosalicilates; } \\
\text { steroids; } \\
\text { thiopurines }\end{array}$ & $\begin{array}{l}28.94 \%(11 / 38)= \\
\text { clinical remission in } \\
2 \text { weeks. } 16 \text {-month } \\
\text { colectomy rate }=34 \%\end{array}$ \\
\hline Hogenauer $^{(13)}$ & 2003 & Austria & $\begin{array}{l}\text { Moderate-to- } \\
\text { severe colitis/ } \\
\text { steroid- } \\
\text { refractory }\end{array}$ & $\begin{array}{l}\text { Truelove Witts } \\
\text { Score. Evaluation up } \\
\text { to week } 12\end{array}$ & 9 & $\begin{array}{l}\text { Tacrolimus } \\
0.15 \mathrm{mg} / \mathrm{kg} / \text { day } \\
\text { (adjusted by serum } \\
\text { target level: } 10-20 \\
\mathrm{ng} / \mathrm{mL} \text { ) }\end{array}$ & $\begin{array}{l}\text { Steroids; } \\
\text { thiopurines }\end{array}$ & $\begin{array}{l}66.66 \%(6 / 9)=\text { clinical } \\
\text { remission in } 12 \text { weeks. } \\
3 / 9=33.33 \% \text { of } \\
\text { colectomy on follow up }\end{array}$ \\
\hline Baumgart $^{(4)}$ & 2006 & Germany & $\begin{array}{l}\text { Steroid- } \\
\text { dependent or } \\
\text { refractory }(57 \% \\
\text { pancolitis) }\end{array}$ & $\begin{array}{l}\text { Modified Clinical } \\
\text { Activity Index up to } \\
30 \text { days }\end{array}$ & 40 & $\begin{array}{l}\text { Tacrolimus } 0.1 \mathrm{mg} / \\
\mathrm{kg} / \text { day (adjusted by } \\
\text { serum target level: } \\
4-8 \mathrm{ng} / \mathrm{mL} \text { ) }\end{array}$ & Steroids & $\begin{array}{l}45 \%(18 / 40)=\text { clinical } \\
\text { remission in } 30 \text { days; } \\
77.5 \%(31 / 40) \text { of } \\
\text { clinical response. } 22.5 \% \\
\text { colectomy on follow up }\end{array}$ \\
\hline $\mathrm{Ng}^{(22)}$ & 2007 & England & $\begin{array}{l}\text { Steroid- } \\
\text { dependent } \\
\text { moderate-to- } \\
\text { sevre colitis or } \\
\text { previous failure } \\
\text { to thiopurines or } \\
\text { Infliximab }\end{array}$ & $\begin{array}{l}\text { Truelove Witts } \\
\text { score up to } 4 \text { weeks }\end{array}$ & 6 & $\begin{array}{l}\text { Tacrolimus } 0.1 \mathrm{mg} / \\
\mathrm{kg} / \text { day (adjusted by } \\
\text { serum target level: } \\
5-10 \mathrm{ng} / \mathrm{mL} \text { ) }\end{array}$ & $\begin{array}{l}\text { Aminosalicilates; } \\
\text { steroids; } \\
\text { thiopurines; } \\
\text { probiotics }\end{array}$ & $\begin{array}{l}50 \%(3 / 6)=\text { clinical } \\
\text { remission in } 4 \text { weeks; } \\
66.66 \%(4 / 6) \text { of clinical } \\
\text { response }\end{array}$ \\
\hline Benson $^{(5)}$ & 2007 & USA & $\begin{array}{l}\text { Steroid- } \\
\text { dependent } \\
\text { or refractory } \\
\text { moderate-to- } \\
\text { severe colitis }\end{array}$ & $\begin{array}{l}\text { Clinical variables } \\
\text { and progression to } \\
\text { colectomy up to } 29 \\
\text { weeks }\end{array}$ & 32 & $\begin{array}{l}\text { Tacrolimus } 0.2 \mathrm{mg} / \\
\mathrm{kg} / \text { day (adjusted } \\
\text { by serum target } \\
\text { level: } 10-12 \mathrm{ng} / \mathrm{mL} \text { ) }\end{array}$ & $\begin{array}{l}\text { aminosalicilates; } \\
\text { steroids; } \\
\text { thiopurines }\end{array}$ & $\begin{array}{l}9.37 \%(3 / 32)=\text { clinical } \\
\text { remission; } 68.75 \% \\
(22 / 32) \text { of clinical } \\
\text { response. } 37.5 \%= \\
\text { colectomy on follow up }\end{array}$ \\
\hline
\end{tabular}

Continue 


\begin{tabular}{|c|c|c|c|c|c|c|c|c|}
\hline Yamamoto $^{(32)}$ & 2008 & Japan & $\begin{array}{l}\text { Moderate-to- } \\
\text { severe colitis } \\
\text { refractory to } \\
\text { other treatments } \\
\text { ( } 81.5 \% \\
\text { pancolitis) }\end{array}$ & $\begin{array}{l}\text { Truelove Witts } \\
\text { Score up to } 30 \text { days }\end{array}$ & 27 & $\begin{array}{l}\text { Tacrolimus } 0.1 \mathrm{mg} / \\
\mathrm{kg} / \text { day (adjusted } \\
\text { by serum target } \\
\text { level: } 10-15 \mathrm{ng} / \mathrm{mL} \text { ) }\end{array}$ & $\begin{array}{l}\text { aminosalicilates; } \\
\text { steroids; } \\
\text { thiopurines }\end{array}$ & $\begin{array}{l}70.4 \%(19 / 27)=\text { clinical } \\
\text { remission in } 30 \text { days; } \\
77.8 \%(21 / 27) \text { of clinical } \\
\text { response. } 26.9 \%= \\
\text { colectomy on follow up }\end{array}$ \\
\hline Thin $^{(30)}$ & 2012 & Australia & $\begin{array}{l}\text { Moderate-to- } \\
\text { severe colitis } \\
\text { refractory to } \\
\text { other treatments }\end{array}$ & $\begin{array}{l}\text { Colitis Activity } \\
\text { Index up to } 30 \text { days }\end{array}$ & 24 & $\begin{array}{l}\text { Tacrolimus } 0.1 \mathrm{mg} / \\
\mathrm{kg} / \text { day (adjusted } \\
\text { by serum target } \\
\text { level: } 8-12 \mathrm{ng} / \mathrm{mL} \text { ) }\end{array}$ & $\begin{array}{l}\text { aminosalicilates; } \\
\text { steroids }\end{array}$ & $\begin{array}{l}37.5 \% \text { clinical remission } \\
(9 / 24) ; 58.3 \%(14 / 24) \\
\text { clinical response }\end{array}$ \\
\hline Schmidt $^{(28)}$ & 2013 & Germany & $\begin{array}{l}\text { Steroid- } \\
\text { refractory } \\
\text { Moderate-to- } \\
\text { severe colitis }\end{array}$ & $\begin{array}{l}\text { Lichtiger score up } \\
\text { to } 12 \text { weeks; need } \\
\text { for colectomy }\end{array}$ & 130 & $\begin{array}{l}\text { Tacrolimus } 0.1 \mathrm{mg} / \\
\mathrm{kg} / \text { day }\end{array}$ & $\begin{array}{l}\text { aminosalicilates; } \\
\text { steroids; } \\
\text { thiopurines }\end{array}$ & $\begin{array}{l}72 \%(94 / 130) \text { clinical } \\
\text { remission; } 14 \% \\
(18 / 130)=\text { colectomy on } \\
\text { follow up }\end{array}$ \\
\hline Inoue $^{(15)}$ & 2013 & Japan & $\begin{array}{l}\text { Moderate-to- } \\
\text { severe pancolitis } \\
\text { without steroid } \\
\text { treatment }\end{array}$ & $\begin{array}{l}\text { Lichtiger score. } \\
\text { Mayo score for } \\
\text { mucosal healing up } \\
\text { to } 4 \text { weeks. }\end{array}$ & 11 & $\begin{array}{l}\text { Tacrolimus } 0.1 \mathrm{mg} / \\
\mathrm{kg} / \text { day (adjusted } \\
\text { by serum target } \\
\text { level: } 10-15 \mathrm{ng} / \mathrm{mL} \text { ) }\end{array}$ & $\begin{array}{l}\text { aminosalicilates; } \\
\text { thiopurines }\end{array}$ & $\begin{array}{l}72.7 \%(8 / 11) \text { clinical } \\
\text { remission; } 100 \%(11 / 11) \\
\text { clinical response }\end{array}$ \\
\hline Miyoshi $^{(21)}$ & 2013 & Japan & $\begin{array}{l}\text { Steroid- } \\
\text { dependent } \\
\text { or refractory } \\
\text { moderate-to- } \\
\text { severe colitis }\end{array}$ & $\begin{array}{l}\text { Lichtiger score. } \\
\text { Mayo score for } \\
\text { mucosal healing up } \\
\text { to } 12 \text { weeks. }\end{array}$ & 51 & $\begin{array}{l}\text { Tacrolimus } \\
\text { initial dose = } 5 \\
\text { mg/day; then, } \\
\text { oral Tacrolimus } \\
\text { (adjusted by serum } \\
\text { target level: } \\
10-15 \mathrm{ng} / \mathrm{mL} \text { ) }\end{array}$ & $\begin{array}{l}\text { aminosalicilates; } \\
\text { steroids; } \\
\text { thiopurines }\end{array}$ & $\begin{array}{l}39.2 \%(20 / 51)=\text { clinical } \\
\text { remission; } 62.74 \% \\
(32 / 51)=\text { clinical } \\
\text { response }\end{array}$ \\
\hline Landy $^{(17)}$ & 2013 & England & $\begin{array}{l}\text { Moderate-to- } \\
\text { severe colitis } \\
\text { refractory to } \\
\text { other treatments }\end{array}$ & $\begin{array}{l}\text { Truelove Witts } \\
\text { Score up to } 6 \\
\text { months }\end{array}$ & 25 & $\begin{array}{l}\text { Tacrolimus } 0.1 \mathrm{mg} / \\
\mathrm{kg} / \text { day (adjusted } \\
\text { by serum target } \\
\text { level: } 5-10 \mathrm{ng} / \mathrm{mL} \text { ) }\end{array}$ & $\begin{array}{l}\text { aminosalicilates; } \\
\text { steroids; } \\
\text { thiopurines }\end{array}$ & $\begin{array}{l}20 \%(5 / 25)=\text { clinical } \\
\text { remission; } 24 \%(6 / 25)= \\
\text { clinical response }\end{array}$ \\
\hline Boschetti ${ }^{(6)}$ & 2014 & France & $\begin{array}{l}\text { Moderate-to- } \\
\text { severe colitis } \\
\text { refractory to } \\
\text { other treatments }\end{array}$ & $\begin{array}{l}\text { UC-DAI at } 4 \text { and } \\
12 \text { weeks }\end{array}$ & 30 & $\begin{array}{l}\text { Tacrolimus } 0.1- \\
0.2 \mathrm{mg} / \mathrm{kg} / \mathrm{day} \\
\text { (adjusted by serum } \\
\text { target level:10-15 } \\
\mathrm{ng} / \mathrm{mL} \text { ) }\end{array}$ & $\begin{array}{l}\text { steroids; } \\
\text { thiopurines }\end{array}$ & $\begin{array}{l}47 \%(14 / 30)=\text { clinical } \\
\text { remission; } 70 \%(21 / 30)= \\
\text { clinical response in } 4 \\
\text { weeks }\end{array}$ \\
\hline Hiraoka $^{(11)}$ & 2015 & Japan & $\begin{array}{l}\text { Colitis } \\
\text { moderada- } \\
\text { severa cortico- } \\
\text { dependiente/ } \\
\text { refractaria }\end{array}$ & $\begin{array}{l}\text { Lichtiger score up } \\
\text { to } 2-3 \text { weeks }\end{array}$ & 47 & $\begin{array}{l}\text { Tacrolimus } 0.05- \\
0.15 \mathrm{mg} / \mathrm{kg} / \text { day } \\
\text { (adjusted by serum } \\
\text { target level: } 10-15 \\
\text { ng/mL) }\end{array}$ & $\begin{array}{l}\text { aminosalicilates; } \\
\text { steroids; } \\
\text { thiopurines }\end{array}$ & $\begin{array}{l}87 \%(41 / 47) \text { showed } \\
\text { remission and/or clinical } \\
\text { response }\end{array}$ \\
\hline Ikeya $^{(14)}$ & 2015 & Japan & $\begin{array}{l}\text { Moderate-to- } \\
\text { severe colitis }\end{array}$ & $\begin{array}{l}\text { Colitis Activity } \\
\text { Index. Mayo score } \\
\text { for mucosal healing } \\
\text { up to } 12 \text { weeks }\end{array}$ & 44 & $\begin{array}{l}\text { Tacrolimus } 0.1 \mathrm{mg} / \\
\mathrm{kg} / \text { day (adjusted } \\
\text { by serum target } \\
\text { level: } 10-15 \mathrm{ng} / \mathrm{mL} \text { ) }\end{array}$ & $\begin{array}{l}\text { aminosalicilates; } \\
\text { steroids; } \\
\text { thiopurines }\end{array}$ & $\begin{array}{l}86.4 \%(38 / 44)=\text { clinical } \\
\text { response; } 65.9 \% \\
(29 / 44)=\text { clinical } \\
\text { remission; } 43.8 \% \\
\text { mucosal healing }\end{array}$ \\
\hline Kawakami $^{(16)}$ & 2015 & Japan & $\begin{array}{l}\text { Steroid- } \\
\text { dependent } \\
\text { or refractory } \\
\text { moderate-to- } \\
\text { severe colitis }\end{array}$ & $\begin{array}{l}\text { Lichtiger score up } \\
\text { to } 4 \text { weeks }\end{array}$ & 49 & $\begin{array}{l}\text { Tacrolimus } 0.1 \mathrm{mg} / \\
\mathrm{kg} / \text { day (adjusted } \\
\text { by serum target } \\
\text { level: } 10-15 \mathrm{ng} / \mathrm{mL} \text { ) }\end{array}$ & $\begin{array}{l}\text { aminosalicilates; } \\
\text { steroids; } \\
\text { thiopurines }\end{array}$ & $\begin{array}{l}89.6 \%=\text { clinical } \\
\text { response; } 75.6 \%= \\
\text { clinical remission in } 4 \\
\text { weeks }\end{array}$ \\
\hline Minami $^{(20)}$ & 2015 & Japan & $\begin{array}{l}\text { Moderate-to- } \\
\text { severe colitis } \\
\text { refractory to } \\
\text { other treatments }\end{array}$ & $\begin{array}{l}\text { Mayo score up to } 8 \\
\text { weeks }\end{array}$ & 22 & $\begin{array}{l}\text { Tacrolimus } 0.1 \mathrm{mg} / \\
\mathrm{kg} / \text { day (adjusted } \\
\text { by serum target } \\
\text { level: } 10-15 \mathrm{ng} / \mathrm{mL} \text { ) }\end{array}$ & $\begin{array}{l}\text { aminosalicilates; } \\
\text { steroids; } \\
\text { thiopurines }\end{array}$ & $\begin{array}{l}90.9 \%(20 / 22)=\text { clinical } \\
\text { response; } 63.63 \% \\
(14 / 22)=\text { clinical } \\
\text { remission in } 8 \text { weeks }\end{array}$ \\
\hline Hiraoka $^{(12)}$ & 2015 & Japan & $\begin{array}{l}\text { Moderate-to- } \\
\text { severe colitis } \\
\text { refractory to } \\
\text { other treatments }\end{array}$ & CAI & 24 & $\begin{array}{l}\text { Tacrolimus } 0.1 \mathrm{mg} / \\
\mathrm{kg} / \text { day (adjusted } \\
\text { by serum target } \\
\text { level: } 10-15 \mathrm{ng} / \mathrm{mL} \text { ) }\end{array}$ & $\begin{array}{l}\text { aminosalicilates; } \\
\text { steroids; } \\
\text { thiopurines }\end{array}$ & $\begin{array}{l}79 \%(19 / 24)=\text { remission } \\
\text { and/or response }\end{array}$ \\
\hline Endo $^{(9)}$ & 2016 & Japan & $\begin{array}{l}\text { Steroid- } \\
\text { dependent } \\
\text { or refractory } \\
\text { moderate-to- } \\
\text { severe colitis }\end{array}$ & CAI up to 8 weeks & 47 & $\begin{array}{l}\text { Tacrolimus } 0.1 \mathrm{mg} / \\
\mathrm{kg} / \text { day (adjusted } \\
\text { by serum target } \\
\text { level: } 10-15 \mathrm{ng} / \mathrm{mL} \text { ) }\end{array}$ & $\begin{array}{l}\text { aminosalicilates; } \\
\text { steroids; } \\
\text { thiopurines }\end{array}$ & $\begin{array}{l}68.08 \%(32 / 47)= \\
\text { clinical response; } \\
55.31 \%(26 / 47)= \\
\text { clinical remission. } \\
14.89 \%(7 / 47)= \\
\text { colectomy on follow up }\end{array}$ \\
\hline Yamamoto $^{(33)}$ & 2016 & Japan & $\begin{array}{l}\text { Steroid- } \\
\text { dependent } \\
\text { or refractory } \\
\text { moderate-to- } \\
\text { severe colitis }\end{array}$ & $\begin{array}{l}\text { UC-DAI up to } 12 \\
\text { weeks }\end{array}$ & 50 & $\begin{array}{l}\text { Tacrolimus } 0.1 \mathrm{mg} / \\
\mathrm{kg} / \text { day (adjusted } \\
\text { by serum target } \\
\text { level: } 10-15 \mathrm{ng} / \mathrm{mL} \text { ) }\end{array}$ & $\begin{array}{l}\text { aminosalicilates; } \\
\text { steroids; } \\
\text { thiopurines }\end{array}$ & $\begin{array}{l}62 \%(31 / 50)=\text { clinical } \\
\text { response; } 40 \%(20 / 50)= \\
\text { clinical remission }\end{array}$ \\
\hline Nuki $^{(23)}$ & 2016 & Japan & $\begin{array}{l}\text { Moderate-to- } \\
\text { severe colitis }\end{array}$ & $\begin{array}{l}\text { UC-DAI up to } 10 \\
\text { weeks }\end{array}$ & 21 & $\begin{array}{l}\text { Tacrolimus } 0.1 \mathrm{mg} / \\
\mathrm{kg} / \text { day (adjusted } \\
\text { by serum target } \\
\text { level: } 10-15 \mathrm{ng} / \mathrm{mL} \text { ) }\end{array}$ & $\begin{array}{l}\text { aminosalicilates; } \\
\text { steroids; } \\
\text { thiopurines }\end{array}$ & $\begin{array}{l}85.7 \%(18 / 21)=\text { clinical } \\
\text { response; } 66.7 \% \\
(14 / 21)=\text { clinical } \\
\text { remission in } 10 \text { weeks }\end{array}$ \\
\hline
\end{tabular}

UC: ulcerative colitis. DAI: disease activity index; CAI: colitis activity index. 
Only two studies ${ }^{(24,25)}$ (Ogata 2006 and Ogata 2012) were randomized controlled trials. The features of these trials are shown in Table 2. Overall, $127 \mathrm{UC}$ patients were included for meta-analysis. Table 3 shows the methodological analysis of these two trials. As witnessed in Figure 2, tacrolimus significantly increased the risk of clinical response versus placebo [RR of clinical response failure $0.58(0.45-0.73)]$, with a NNT of 3 . Figure 3 highlights the $\mathrm{RR}$ of failure of clinical remission versus placebo, which was 0.91 $(0.82-1)$, with a NNT of 10. Mucosal healing, as shown in Figure 4 , was also significantly favoured by tacrolimus treatment [RR 0.59 $(0.46-0.74)]$ with a NNT of 3 . According to Egger test, a low risk of publication bias was found $(P>0.5)$.

TABLE 2. Characteristics of randomized controlled trials included in meta-analysis

\begin{tabular}{|c|c|c|c|c|c|c|c|}
\hline Author & Year & Country & $\begin{array}{l}\text { UC severity and } \\
\text { distribution }\end{array}$ & $\begin{array}{l}\text { Remission definition (and } \\
\text { time considered) }\end{array}$ & Number of patients & Interventions & $\begin{array}{c}\text { Concomitant } \\
\text { treatment }\end{array}$ \\
\hline
\end{tabular}

UC: ulcerative colitis. DAI: disease activity index.

TABLE 3. Methodological analysis of randomized controlled trials included in meta-analysis

\begin{tabular}{|c|c|c|c|c|c|c|}
\hline ID Study & $\begin{array}{l}\text { Concealed random } \\
\text { allocation }\end{array}$ & $\begin{array}{l}\text { Blinding of patients } \\
\text { and medical team }\end{array}$ & $\begin{array}{c}\text { Similar interventions } \\
\text { between groups }\end{array}$ & $\begin{array}{l}\text { Complete } \\
\text { follow up }\end{array}$ & $\begin{array}{l}\text { Intention to } \\
\text { treat analysis }\end{array}$ & Jadad score \\
\hline Ogata $2006^{(25)}$ & Not clear & Yes & Yes & Yes & No & 4 \\
\hline
\end{tabular}

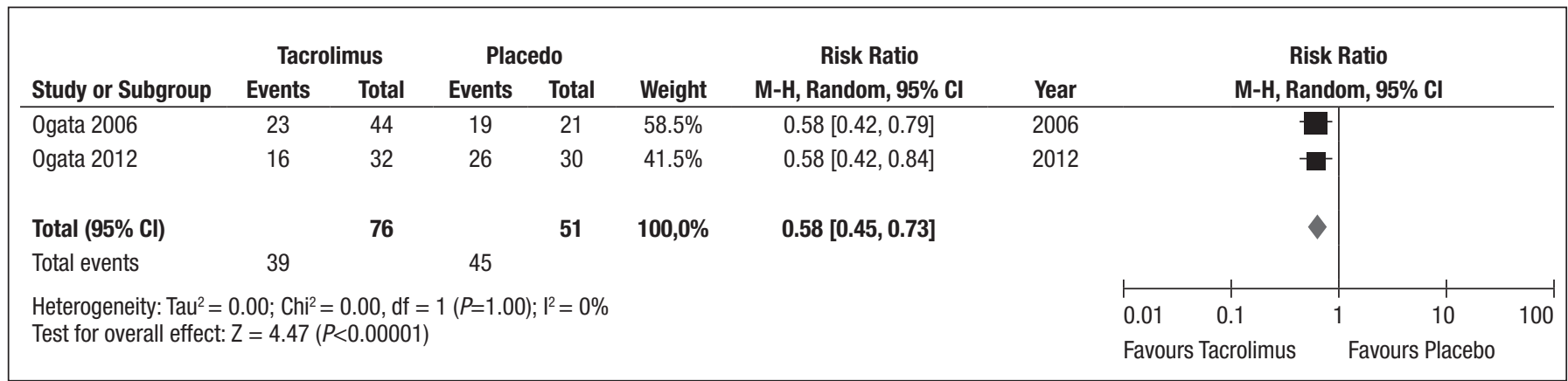

FIGURE 2. Forest Plot showing meta-analysis on clinical response of tacrolimus versus placebo.

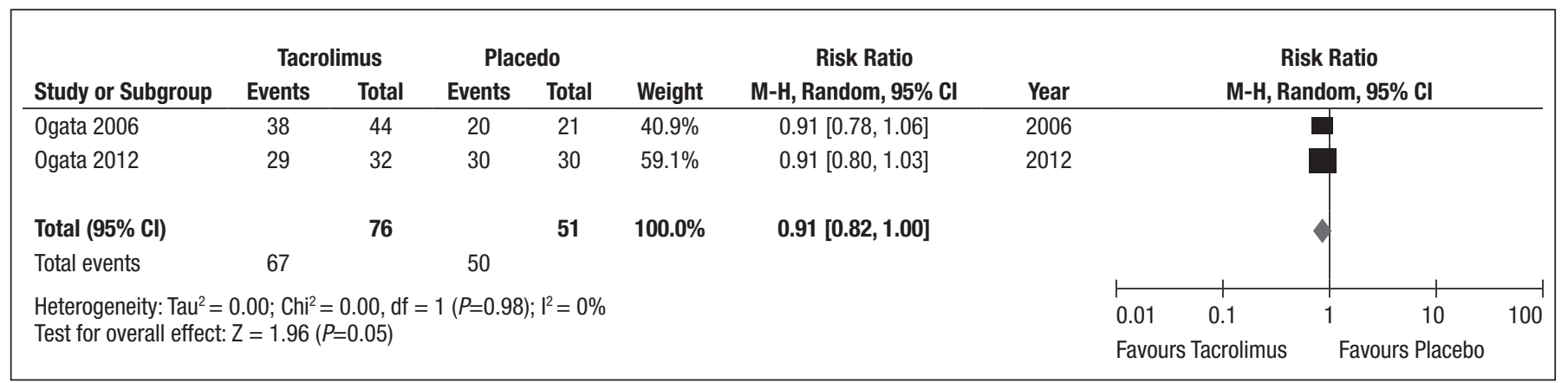

FIGURE 3. Forest Plot showing meta-analysis on clinical remission of tacrolimus versus placebo. 


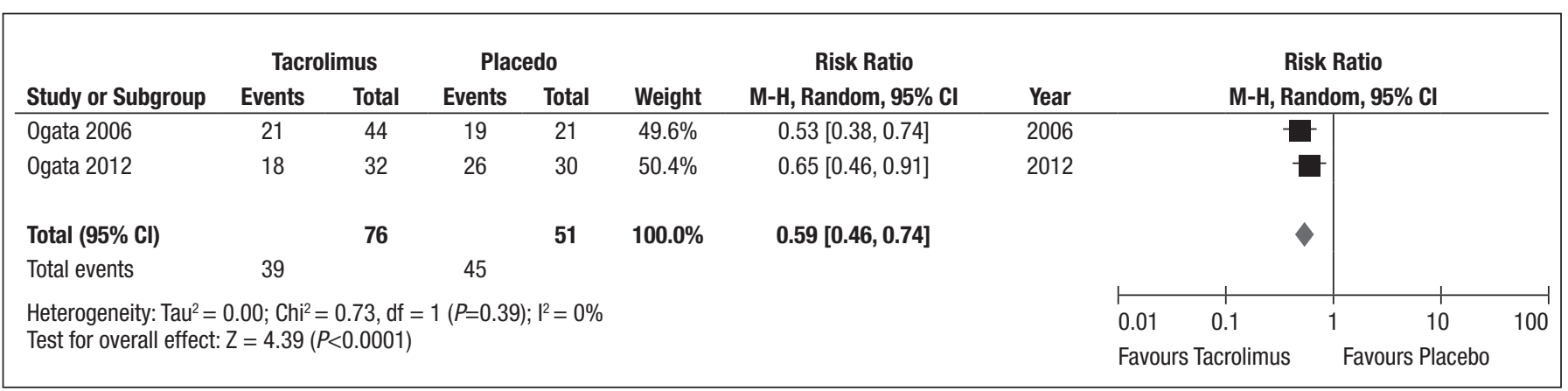

FIGURE 4. Forest Plot showing meta-analysis on mucosal healing of tacrolimus versus placebo.

The most commonly reported severe adverse events were gastroenteritis and sepsis. When considering only the randomized controlled trials, there was not a significant increase in the risk of serious adverse events versus placebo [RR 3.38 (0.17-68.91)].

\section{DISCUSSION}

According to the results observed in our systematic review, tacrolimus therapy could be a valid alternative in the context of moderate-to-severe UC that have not responded to other treatments or who are regarded as cortico-dependent or refractory.

As mentioned before, evidence on the efficacy of calcineurin inhibitors derives mainly from the experience of cyclosporine as a rescue therapy in the setting of severe UC. In a pioneer study, Lichtiger et al. ${ }^{(18)}$ showed that endovenous cyclosporine was useful to avoid colectomy in patients with severe UC flares after systemic steroid therapy failure. D'Haens et al. ${ }^{(8)}$ concluded that cyclosporine is a valid alternative to intravenous steroids in severe flares. Additionally, controlled studies have shown that cyclosporine may be equivalent to Infliximab in this type of clinical scenario ${ }^{(18)}$. Nevertheless, it should be reminded that cyclosporine has a nonneglectable adverse event profile and there is much less evidence apart from its parenteral use.

Tacrolimus is a well-known calcineurin inhibitor that is widely used as immunosupresant therapy in the context of transplant or even for the treatment of autoinmmune conditions ${ }^{(31)}$. Tacrolimus has the advantage that it can be administered orally and that its serum concentration can be easily measured and adjusted to reach the adequate level in each case. The initial experience in UC patients was originated in referral centers from Japan, and then it was adopted by some centers in Europe. According to what is witnessed in uncontrolled studies, tacrolimus shows a high proportion of clinical response in the short-term, with a low NNT; however, when clinical remission is considered, such proportion is lower and more variable.

It is worth mentioning, according to what is observed from uncontrolled studies, the relatively high proportion of patients who would eventually require colectomy in spite of receiving tacrolimus $^{(4,5,10,13,28,32)}$ (Fellermann 2002, Hogenauer 2003, Baumgart 2006, Benson 2007, Yamamoto 2008, Schmidt 2013). As a consequence, it would seem like tacrolimus could delay the need for colectomy in a selected group of patients - those moderate-to-severe UC patients with prior failure to other therapeutic alternatives. It should also be mentioned that no study has considered tacrolimus therapy in patients with less severe disease.

There is a scarcity of high-quality trials to know the real impact of tacrolimus in the management of UC. As a matter of fact, as shown by this review, there has only been published two controlled trials ${ }^{(24,25)}$ (Ogata 2006 and Ogata 2012), with a relatively low number of patients and a rather short follow up time. One strength of these two trials is that mucosal healing was included as an outcome, which is known to be a relevant prognostic factor and a therapeutic target, particularly when it comes to UC ${ }^{(26)}$. It is also worth mentioning that all studies assessed the efficacy of oral tacrolimus for the continued treatment of UC patients.

Evidence on the efficacy of calcineurin inhibitors in the setting of CD is more scarce: there are only a few controlled trials, mainly on patients with perianal disease, and few cohort studies ${ }^{(19)}$. Nevertheless, the relatively few alternatives in severe CD may turn tacrolimus into a valid therapeutic option, regardless of the few scientific evidence available.

Some limitations should be mentioned. First of all, there were few studies included for meta-analysis, so the conclusions should be cautiously interpreted. We decided not to perform meta-analysis with observational uncontrolled studies, because this represents a high risk for relevant biases, which could in turn produce a distortion in the conclusions derived from higherquality studies. Last but not least, it is worth mentioning that most observational studies were retrospective, with the logical limitations that this implicate.

In conclusion, tacrolimus seems to show in both uncontrolled studies as well as in placebo controlled trials, a significant efficacy to induce clinical response and remission in moderate-to-severe UC. However, more evidence is undoubtfully needed to fully estimate the magnitude of its benefit.

\section{Authors' contributions}

Juan Lasa: bibliographic review, data analysis, statistical analysis, manuscript review. Pablo Olivera: bibliographic review, data analysis, manuscript elaboration. 
Lasa J, Olivera P. Eficácia do tacrolimus para indução de remissão em pacientes com colite ulcerosa moderada a grave: uma revisão sistemática e meta-análise. Arq Gastroenterol. 2017;54(2):167-72.

RESUMO - Contexto - Há evidências que mostram que os inibidores de calcineurina podem ser úteis para o tratamento da colite ulcerativa severa. No entanto, há poucos dados sobre a eficácia do tacrolimus para indução de remissão neste cenário. Objetivo - Desenvolver uma revisão sistemática sobre evidências existentes sobre a eficácia clínica do tacrolimus para a indução de remissão em pacientes com colite ulcerosa de moderada a grave. Métodos - Realizada pesquisa bibliográfica de 1966 a agosto de 2016 usando MEDLINE, Embase, LILACS e Biblioteca Cochrane. Foram utilizados os seguintes termos MeSH: "doenças inflamatórias intestinais" ou "colite ulcerativa "e "inibidores da calcineurina" ou "tacrolimo" ou "FK506". Foram considerados para revisão estudos que avaliaram a eficácia clínica do tacrolimus para a indução de remissão em pacientes adultos com colite ulcerosa. Uma meta-análise foi realizada com esses estudos incluídos que também fossem controlados por placebo e randomizados. Avaliou-se a resposta clínica, bem como remissão clínica e a cicatrização da mucosa. Resultados - No total, 755 referências foram identificadas, dos quais 22 estudos foram finalmente incluídos. Apenas dois deles eram experimentações randomizadas e, placebo-controlada. Um total de 172 pacientes foram avaliados. Verificou-se um risco significativamente menor de falha na resposta clínica para tacrolimus versus placebo [RR 0.58 (0.45-0,73)]; Além disso, um menor risco de falha na indução da remissão também foi encontrado versus placebo [RR 0,91 (0,82-1)]. Conclusão - Tacrolimus parece ser uma alternativa terapêutica válida para a indução de remissão em pacientes com colite ulcerosa moderada a grave.

DESCRITORES - Doenças inflamatórias intestinais. Tacrolimo. Inibidores de calcineurina.

\section{REFERENCES}

1. Abraham C, Cho JC. Inflammatory bowel disease. N Engl J Med. 2009;361:2066-78

2. Actis GC, Volpes R, Rizzetto M. Oral microemulsion cyclosporine to reduce steroids rapidly in chronic active ulcerative colitis. European J Gastroentero Hepatol. 1999;11:905-8

3. Allez M, Karmiris K, Louis E, et al. Report of the ECCO pathogenesis work shop on anti-TNF therapy failures in inflammatory bowel diseases: Definitions, frequency and pharmacological aspects. J Crohn's Colitis. 2010;4:355-66.

4. Baumgart DC, Pintoffl J, Sturm A, Wiedenmann B, Dignass AU. Tacrolimus is safe and effective in patients with severe steroid-refractory or steroid-dependent inflammatory bowel disease- a long-term follow up. Am J Gastroenterol. 2006;101:1048-56.

5. Benson A, Barrett T, Sparberg M, Buchman AL. Efficacy and safety of tacrolimus in refractory ulcerative colitis and crohn's disease: a single center experience. Inflamm Bowel Dis. 2008;14:7-12.

6. Boschetti G, Nancey S, Moussata D, Stefanescu C, Roblin X, Chauvenet M, Stroeymeyt K, Bouhnik Y, Flourie B. Tacrolimus induction followed by maintenance monotherapy is useful in selected patients with moderate-to-severe ulcerative colitis refractory to prior treatment. Dig Liv Dis. 2014;46:875-80.

7. Costa J, Magro F, Caldeira D, Alarcão J, Sousa R, Vaz-Carneiro A. Infliximab Reduces Hospitalizations and Surgery Interventions in Patients With Inflammatory Bowel Disease: A Systematic Review and Meta-analysis. Inflamm Bowel Dis. 2013;19:2098-110.

8. D'Haens G, Lemmens L, Geboes K, Vandeputte L, Van Acker F, Mortelmans L, Peeters M, Vermeire S, Penninckx F, Nevens F, Hiele M, Rutgeerts P. Intravenous cyclosporine versus intravenous corticosteroids s single therapy for severe attacksof ulcerative colitis. Gastroenterology. 2001;120:1323-9.

9. Endo K, Onodera M, Shiga H, Kuroha M, Kimura T, Hiramoto K, Kakuta Y, Kinouch Y, Shimosegawa T. A comparison of short nd long-term therapeutic outcomes of infliximab - versus tacrolimus-based strategies for steroid-refractory ulcerative colitis. Gastroenterol Res Prac 2016;3162595.

10. Fellermann K, Tanko Z, Herrlinger KR, Witthoeft T, Homann N, Bruening A, Ludwig D, Stange EF. Response of refractory colitis to intravenous or oral tacrolimus (FK506). Inflamm Bowel Dis. 2002;8:317-24

11. Hiraoka S, Kato J, Moritou Y, Takei D, Inokuchi T, Nakarai A, Takahasi S, Harada K, Okada H, Yamamoto K. The earliest trough concentrarionredicts the dose of tacrolimus required for remission induction therapy in ulcerative colitis patients. BMC Gastroenterol. 2015;15:53.

12. Hiraoka S, Inokuchi T, Takei D, Nakarai A, Sugihara Y, Morito Y, Takahashi S, Harada K, Okada H, Yamamoto K, Kato J. Tacrolimus therapy for outpatients with refractory ulcerative colitis. Gastroenterology. 2015;148:(Suppl 1):870-1

13. Hogenauer $\mathrm{H}$ et al. Effect of oral tacrolimus (FK506) on steroid-refractory moderate/severe ulcerative colitis. Aliment Pharmacol Ther. 2003;18:415-23.

14. Ikeya K, Sugimoto K, Kawasaki S, Iida T, Maruyama Y, Watanabe F, Hanai H. Tacrolimus for remission inductionn ulcerative colitis: Mayo endoscopic subscore 0 and 1 predicts long-term prognosis. Dig Liv Dis. 2015; 47:365-71.

15. Inoue T, Murano M, Narabayashi K, Okada T, Nouda S, Ishida K, et al. The efficacy of oral tacrolimus in patients with moderate/severe ulcerative colitis not receiving concomitant corticosteroide therapy. Intern Med. 2013;52:15-20.

16. Kawakami K, Inoue T, Murano M, Narabayashi K, Nouda S, Ishida K, et al. Effects of oral tacrolimus s a rapid induction therapy in ulcerative colitis. World J Gastroenterol. 2015;21:1880-6.

17. Landy J, Wahed M, Peake STC, Hussein M, Ng SC, Lindsay JO, Hart AL. Oral tacrolimus s maintenance therapy for refractory ulcerative colitis - an analysis of outcomes in two London tertiary centers. J Crohn Colitis. 2013;7:e516-e521
18. Lichtiger S, Present DH, Kornbluth A, Gelernt I, Bauer J, Galler G, Michelass M, Hanauer S. Cyclosporine in severe ulcerative colitis refractory to steroid therapy. N Engl J Med. 1994;330:1841-5.

19. McSharry K, Dalzell AM, Leiper K, El-Matary W. Systematic review: the role of tacrolimus in the management of Crohn's disease. Aliment Pharmacol Ther. 2011;34:1282-94

20. Minami N, Yoshino T, Matsuura M, Koshikawa Y, Yamada S, Toyonaga T, Madian A, Honzawa Y, Nakase H. Tacrolimus or infliximab for severe ulcerative colitis: short-term and long-term data from a retrospective observational study. BMJ Open Gastro. 2015;2:e00021

21. Miyoshi J, Matsuoka K, Inoue N, Hisamatsu T, Ichikawa R, Yajima T, Okamoto S, Naganuma M, Sato T, Kanai T, Ogata H, Iwao Y, Hibi T. Mucosal healing with oral tacrolimus is associated with medium-nd long-term prognosis in steroid-refractory/dependent ulcerative colitis patients. J Crohn Colitis. 2013;7:e609-e614

22. Ng SC, Arebi N, Kamm MA. Medium-term results of oral tacrolimus treatmentin refractory inflammatory bowel disease. Inflamm Bowel Dis. 2007;3:129-34.

23. Nuki Y, Esaki M, Asano K, Maehata Y, Umeno J, Moriyama T, Nakamura S, Matsumoto T, Kitazono T. Comparison of the therapeutic efficacy and safety between tacrolimus and infliximab for moderate-to severe ulcerative colitis: single center experience. Scand J Gastroenterol 2016;51:700-5.

24. Ogata H, Kato J, Hirai F, Hida N, Matsui T, Matsumoto T, Koyanagi K, Hibi T. Double-blind, placebo controlled trial of oral tacrolimus (FK506) in the management og hospitalized patients with steroid-refractory ulcerative colitis. Inflamm Bowel Dis. 2012:18:803-8.

25. Ogata H, Matsui T, Nakamura M, Lida M, Takazoe M, Suzuki Y, Hibi T. A randomised dose finding study of oral tacrolimus (FK506) therapy in refractory ulcerative colitis. Gut. 2006;55:1255-62.

26. Pineton de Chambrun G, Peyrin-Biroulet L, Lémann M, Colombel JF. Clinica implications of mucosal healing for the management of IBD. Nat Rev Gastroenterol Hepatol. 2010;7:15-29.

27. Roda G, Jharap B, Neeraj N, Colombel JF. Loss of Response to Anti-TNFs: Definition, Epidemiology, and Management. Clin Transl Gastroenterol. 2016;7:e135.

28. Schmidt KJ, Herrlinger KR, Emmrich J, Barthel D, Koc H, Lehnert H, Stange EF, Fellermann K, Buning J. Short-term efficacy of tacrolimus in steroid-refractory ulcerative colitis - experience in 130 patients. Aliment Pharmacol Ther. 2013;37:129-136

29. Schoenfeld P, Cook D, Hamilton F, Laine L, Morgan D, Peterson W. An evidence-based approach to gastroenterology therapy. Gastroenterology. 1998; $114: 1318-25$

30. Thin LW, Murray K, Lawrance IC. Oral tacrolimus for the treatment of refractory inflammatory bowel diseasein the biologic era. Inflamm Bowel Dis $2013 \cdot 19 \cdot 1490-8$

31. Webster A, Woodroffe RC, Taylor RS. Tacrolimus versus cyclosporin as primary immunosupresion for kidney transplants recipients. CDS Review. 2005;4:CD003961

32. Yamamoto S, Nakase H, Mikami S, Inoue S, Yoshino T, Takeda Y, et al. Longterm effect of tacrolimus therapy in patients with refractory ulcerative colitis. Aliment Pharmacol Ther. 2008;28:589-97.

33. Yamamoto T, Shimoyama T, Umegae S, Matsumoto K. Tacrolimus vs anti-tumor necrosis factor agents formoderately to severely active ulcerative colitis: a retrospective observational study. Aliment Pharmacol Ther. 2016;43:705-16. 